\title{
La Salud Pública al Final del Siglo XX
}

\author{
CARLOS BUSTÍOS \\ Deparamenso de Medicina Preventiva y Salud Pública. Faculuad de Medicina - UNMSM.
}

\begin{abstract}
RESUMEN
La Salud Pública se define como una respuesta colectiva, que se explica como parte de una totalidad histórico-social. En su cvolución después de la II Guerra Mundial, se distinguen dos fases de orientación contraria. Con la crisis de los 70 , los gobiernos del mundo iniciaron reformas de sus sistemas de salud, y al final del Siglo $\mathrm{XX}$, los rasgos de la sociedad occidental validan la teoría de la modernidad de Habermas. En este contexto, se verifica una práctica de la Salud Pública subordinada a una política económica, eje central del proyecto de desarrollo dominante. Práctica que aumenta su eficiencia técnica, pero también las desigualdades en el acceso a los servicios de salud de calidad y en la satisfacción del usuario; hechos éstos derivados esencialmente de la aplicación autoritaria de una racionalidad instrumental que percibe al Sector Salud como una área directa de acumulación económica, antes que de capitalización social de vida saludable. Actualmente, crece el consenso sobre la urgencia de replantear el actual proyecto de desarrollo. Finalmente, una nueva propuesta de reforma de la Salud Pública -que pretenda ser eficaz, correcta y veraz - debe ser resultado de un esfuerzo democrático e interdisciplinario, acorde con Ios Derechos Humanos, y que permita acuerdos propios de una racionalidad comunicativa.
\end{abstract}

Palabras claves: Salud Pública; Planificación en Salud; Racionalización; Reforma en Salud.

\section{THE PUBLIC HEALTI AT THE END OF THE TWENTIETH CENTURY}

\section{SUMMARY}

The term Public Health involves a collective response inside a whole social-historic setting. It was developed after the II World War and had two different phases. With the 70 s crisis, a number of Health Systems reforms were started by governments and at the end of the 20th century, occidental society validates the Habermas theory of the Modernity. In that sense, Public Health Practice is allocated as an economic policy subordinate, being the economic issue the axis of the current development project. This kind of practice improves the technical efficacy, but increases differences in the high-quality health care services access and user dissatisfactions; both of which come essentially from an authoritarianism of a instrumental rationality. That policy considered the Health Sector as a resource-generating being, instead of a good social health producer. Furthermore, there is a growing joint about the urgency to set up new approaches for the current development project. Finally, we considered that any new reform proposed (that attempts to be effective, correct and reliable) must come from an interdisciplinary and democratic effort according with the Human Rights, in order to achieve real agreements.

Keywords: Public Health; Health Care Reform; Health Care Rationing; Reform in Health.

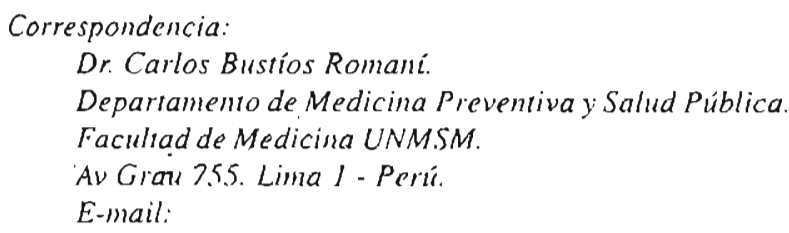




\section{SALUD PÚBLICA Y SOCIEDAD}

\section{El Hombre como un Ser Biosocial}

Mario Bunge ('), distingue tres enfoques ontológicos sobre el hombre:

a. Individualismo. Sólo hay individuos, la sociedad es sólo un conglomerado de individuos.

b. Colectivismo. El individuo es sólo un elemento de una colectividad humana. No es nada fuera de lá colectividad, "el pueblo lo es todo".

c. Sistenismo. Todo individuo forma parte de uno o mas sistemas sociales; "nosotros construinos, mantenemos y reformamos la sociedad, yésta nos forma".

Para los fines de estas notas, asumimos el "sistemismo" y. en consecuencia, caracterizamos al hombre como un ser biológico, social, racional, con sentimientos y valores morales. que existe en un contexto que lo condiciona y es condicionado por él.

El hombre. en tanto es un ser biológico, tiene que satisfacer sus necesidades básicas para sobrevivir y. en tanto es un ser social, debe satisfacer sus necesidades socioculturales y tratar de lograr sus aspiraciones para posibilitar su bienestar general. Los medios para la satisfacción de sus necesidades y el logro de sus aspiraciones se encuentran o se producen socialmente en su contexto. Por eilo, la característica esencial del hombre es su capacidad para la acción racional, a través de la cual transforma o pretende transfommar su contexto en función de sus necesidades y aspiraciones.

La historia del hombre nos muestra que toda la vida social responde a y deriva a ciertas necesidades y aspiraciones humanas, y que todas las acciones sociales para sat isfacerlas o lograrlas están fuertemente intertelacionadas y dependen unas de otras. Esta misma historia, desde sus inicios. nos informa de la existencia de un comportamiento del hombre que es compatible con un conocimiento intuitivo de que el cuidado de su salud es una de sus necesidades básicas. El hombre intuyó y, luego, verificó de que sólo la vida saludable podía garantizar su supervivencia y posibilitar su desarrollo como persona humana. En las últimas décadas, la creciente importancia social asignada a una vida humana saludable, en armonía con la naturaleza y con el resto de los hombres, ha dado nrigen, además, a una nueva aspiración: Ja "salud óprima en un ambiente sustentable". La respuesta social orientada directamente a la satisfacción de esa necesidad y al logro, más recientemente, de esta aspiración es denominada SALUD PÚBLICA $\left({ }^{2}\right)$.

\section{La Salud Pública como Respuesta Social}

La Salud Pública como respuesta social particular puede ser descrita de tres maneras distintas pero complementarias: (a) una práctica social particular, o conjunto de acciones sociales e instrumentales, a través de las cuales se organizan y dirigen los esfuerzos colectivos para la promoción, protección y recuperación de la salud individual y colectiva, en un contexto determinado: (b) un sistema social particular, encargado de la producción de aquellas acciones; $y$ (c) un saber interdisciplinario, o cuerpo de proposiciones verificables y de normas vigentes que fundamentan y legitiman la racionalidad de esa práctica.

La Salud Pública, al igual que toda respuesta social, tiene un carácter histórico social. Social, no solamente en cuanto es una práctica colectiva, sino en cuanto es parte de la sociedad en que se desarrolla y, en consecuencia, está sometida a las mismas determinaciones y a la mismas relaciones que explican el cambio del todo social. Histórica, no sólo en cuanto alude a los cambios de sus límites, contenidos y posibilidades en el liempo, sino a la determinación final de estos cambios por el desarollo paralelo de "lo económico" y "lo político". En cada sociedad concreta. la práctica de la Salud Pública es delimitada, definida y denominada de manera diferente, de acuerdo a un conjunto de criterios cognitivos, culturales, económicos y políticos dominantes o hegemónicos en un determinado período de su particular historia; no obstante, uno puede distinguir algunos rasgos permanentes que le otorgan su idencidad: (a) su objeto: la salud-enfermedad de la población, en otros términos, la salud-enfermedad del público; (b) su fin: la defensa de la vida saludable de la población; (c) su prioridad: la atención de los problemas públicos, antes que los individuales; (d) su conducción: a cargo de la autoridad política o la que representa los intereses comunes de la colectividad; (e) sus líneas de acción: sobre la población y sobre el espacio compartido por la misma $\left({ }^{2}\right)$.

\section{Estrategias Teóricas en Ciencias Sociales}

La función de una estrategia teórica es proporcionar un marco conceptual para describir los rasgos esenciales de un segmento o un aspecto de la realidad que nos interesa conocer y para desarrollar hipótesis de trabajo que lo expli- 
quen. Por el carácter histórico social de la Salud Pública, debemos optar por una estrategia que nos permita conocer, en una primera instancia, la totalidad social o sociedad de la cual forma parte. Para estos fines, optaremos la propuesta que, al respecto, ha planteado Habermas $\left({ }^{3}\right)$.

Desde la perspectiva de la sociología comprensiva, la sociedad es coextensiva al mundo de la vida; es decir, al mundo cotidiano construido por los individuos a partir de las tradiciones culturales comunes y que es caracterizado por la percepción directa, la práctica material y la sociabilidad. Es el mundo de la "integración social", donde lo que vincula a los individuos socializados y asegura la integración de la colectividad es una red de acciones comunicativas de cooperación, orientadas al entendimiento.

De manera distinta, desde la perspectiva de la sociología sistémica, la sociedad es un sistema social que se integra no solamente a través de acciones orientadas al entendimiento, sino también a través de nexos funcionales de cooperación y de control que no son pretendidos y que la mayoría de veces no resultan perceptibles dentro del horizonte del "mundo de la vida". En las sociedades modernas, los mecanismos más importantes de control social o de "regulación no normativa" son el mercado (lo económico) yel Estado(lo político). Por ejemplo, el mercado es uno de los mecanismos de "integración sistémica" que estabiliza redes de acción no pretendidas mediante un entrelazamiento funcional de las "consecuencias" económicas de la acción, mientras que el mecanismo de entendimiento se limita a armonizar entre sí las "orientaciones" de acción de los participantes.

Por lo expuesto, Habermas propone distinguir entre "inregración social" e "integración sistémica": la primera se centra en las orientaciones socionormativas de la acción, en tanto que la segunda opera atravesando dichas orientaciones. En el primer caso, el conjunto de acciones queda integrado a través de un consenso asegurado normativamente, o por medio de un consenso alcanzado por el diálogo; en el segundo, por medio de un control no normativo de decisiones particulares carentes subjetivamente de coordinación. Además, Habermas advierte que si entendemos la integración de la sociedad exclusivamente como "integración social" estamos optando por una estrategia conceptual que concibe a la sociedad como equivalente al mundo de la vida. aceptando tres ficciones: (a) la autonomía de los actores; (b) la independencia de la cultura; y (c) la transparencia del diálogo o comunicación. Pero si, por otro lado, entendemos la integración de la sociedad exclusivamente como "integración sistémica", estamos optando por otra estrategia conceptual, también limilada, que presenta a la sociedad según el modelo de un sistema autorregulado. es decir un tipo especial de sistema biológico.

Para reducir esas limitaciones teóricas, Habermas utiliza los conceptos de "mundo de la vida" y de "sistema" como elementos polares de su concepción de sociedad y, más propiamente, de un orden social que responde a las dos formas de integración descritas. En una primera aproximación, define a la sociedad como: "Un sistema que tiene que cumplir las condiciones de mantenimiento propias de los mundos socioculturales de la vida" o "Plexos de acción sistemáticamente establecidos de grupos integrados sociononnativamente". Precisando, que "la sociedad es una entidad que en el curso de su evolución se diferencia lo mismo como sistema que como mundo de la vida" ( $\left.{ }^{3}\right)$.

\section{Tipología de la Acción}

En tanto existen múltiples conceptos de "acción" que. casi siempre implícitamente, se emplean en teoría sociológica, es conveniente también optar por una tipología que nos permita distinguir los distincos tipos de acción en el ámbito de la Salud Pública. En una primera clasificación de dichas acciones se distinguen:

a. La "acción instrumental". Referida a un "objeto" físico o biológico presente en el mundo objerivo, con el cual hay un simple coexistir. Acción orientada a un fin tecnológico, que busca manipular el objeto. No hay reciprocidad. Se establece una relación sujeto-objeto unívoca. Para garantizar la eficacia de este tipo de acción se utiliza una racionalidad medio-fin, puramente instrumental.

b. La "acción social". Referida a otra persona con la cual se interacciona. Está orientada a condicionar el comportamiento del "otro", a través de una relación sujetocosujeto. Para Habermas todas las referencias a la acción social pueden reducirse en lo esencial a 4 conceptos básicos, que analíticamente es menester distinguir con cuidado: la teleológica, la regulada por normas, la dramatúrgica y la comunicativa.

La acción teleológica, es la desarrolladá por un actor que realiza un fin o hace que se produzca un estado de cosas deseado eligiendo, en una situación dada, los medios más pertinentes y aplicándolos de manera adecuada. Lo central es una decisión entre alternativas de acción, enderezada a 
la realización de un tinn, dirigida pror reglas técnicas y aproyada en una interpretación de la situación. Concepto utilizado por los fundadores de la economía politica neoclásica para desarrollar la teoria de lat decisión económica (†).

La acción estratégica es una moclalidad de la acción teleológica amplialda. (que resultactando, en el cálculo que el actor hace de su éxilo interviene la expectatival de decisiones de a lo menos onc) actor que rambién actúa con vistas a la realización de sus propios fines. Este modelo de acción es interpretado en forma "utilitarista". bajo el supuesto que el acior elige y calcula medios y fines desde el punto de vista de la maximización de utilidades o de expecrativas de utilidad. Fil modelo subyace a los planteamientos que en términos de la "Teoría de los Juegos" se hace en Economía, Sociología y Psicología Social. El resultado de la acción depende rambién de otros actores, cada uno de los cuales se orientia a la consecución de sus fines, y sólo se comporta cooperativamente en la medida de que ello es concordante con su cálculo egocénerico de utilidades ( $\left.{ }^{4}\right)$.

La acción regulada por normas, se refiere al componamiento de los miembros de una colectividad que orientan su acción por valores comunes que se expresan en normas vigentes en esta colectividad. El actor particular observa (cumple) una norma (o la viola) tán pronto que en una situación dada se dan las circunstancias para que la norma se aplique. Las nomas expresan un acuerdo existente en un crupo social: todos los miembros de un grupo para los que rige una delemninada norma tienen derecho a esperar unos de otros su cumplimiento. El concepto centrall es la observancia de una norma, que significa el cumplímiento de una expectativa generalizada de comportamiento. El modelo normativo de acción es el que subyace a la "Teoría del Rol Social", que adquirió un significado paradiggmático en la s(xiología de Durkheim y Parsons $\left({ }^{+}\right)$.

La acción comunicativa se refiere a la interacción de actores que buscan entenderse sobre una situación de acción para poder así coordinar de común acuerdo sus planes particularcs de acción y, de esta manera, sus acciones. El concepto central es el de interprctación, que se refiere primordialmente a la negociación de los aspectos de la sisuación suceptibles de consenso. La acción comunicativa es un modelo orientado para el entendimiento mutuo. El "esicendimiento" es el proceso de obrención de un acuerdo entre sujetos lingüística e interactivamente comperentes; acuerdo que se apoya en conviciones commes. alcanzadas a rravés del diálogo $\left({ }^{+}\right)$.

\section{Racionalidad de Ia Acción}

Para los teóricos de la acción la razón humana se expresa o está encarnada en:

a EI CONOCIMIENTO verdadero, validado por la Teoría la Ciencia y lá Lógica;

b. EI HABLA correcto, validado por la Semíntica y la Linguística: $y$

c. La ACCIÓN eficaz y recta (justal). validada por el discurso tecricico de la Tecnología, y el discurso prácticomoral del Derecho y de la F́tica.

De acuerdo a J. Habermas ( ${ }^{2}$ ), la racionalidad de la acción tiene tres dimensiones y según se enfatice una de ellas o sean consideradas de manera equilibrada se pueden distinguir cuatro tipos de racionalidad:

a. Racionalidad medio-fin. Racionalidad instrumental o cognitivo-instrumental. Orientada al éxito. Trata sobre la racionalidad de la selección de los medios en relación a los fines previamente dados. Se mide por la eficacia de la acción en la producción prevista de estados de cosas en el mundo objetivo o en el condicionamiento de la conducta del "otro" en el mundo social, y por la eficiencia en la normatización técnica de los medios. El análisis del costo-beneficio es su máxima de decisión predominante. El actor social utiliza el lenguaje para influenciar sobre el "oyenle" u "oponente". para lograr efectos imperativos. Este aspecio de racionalidad es enfatizaldoen la acción teleologica instrumental y en la acción estratégica. En Salud Pública. la acción técnica y. muchas veces, la acción administrativa y política pueden presentarse como casos límites de esos tipos de acción, por los cuales el actor social se relaciona con el "otro", como si éste fuera un objeto, su aliado o su rival.

b. Racionalidad valorativa o Comección normaliva. Orientada al entendimiento. Medida por la rectitud en el cumplimiento de las normas vigentes en el mundo social o la corrección de las mismás normas. Jil actor utilizil el lenguaje para el establecimiento de relaciones interporsonales, para lograr efecros regulatorios. Esta racionalidad "valorativa" se constituye rigurosamente. cuando algunas normas éticas penetran sisteınáticamente en el mundo de la vida, adquiriendo una fuerza unificadora de la acción. Este aspecto de la racionalidad es enfatizado en la acción regulada por normas. En Salud Pública, este tipo de acción conespondería a las acciones administrativas y 
técnicas orientadas por los valores éticos vigentes en la colectividad donde se desarrollan.

c. Adecuada autopresentación del mundo interno del actor. Orientada al entendimiento. Medida por lá veracidad de la autopresentación. El actor utiliza el lenguaje para la presentación de sí nismo, para expresarse a.los "orros". Esic aspecto de la racionalidad es enfatizado en la acción dramatúrgica.

d Racionalidad comunicativa. (rientada al entendimiento. Es la que calacteriza a la acción comunicativa. en la cual el acior utiliza las tres dimensiones de la racionalidad, de manera equilibrada. buscando la eficacia. garantizando su lectitud y tratando de ser velaz ("rransparente"o "curémico"). En Salud Pública serían las acciones realizadas con fines. medios y valores consensuales.

\section{LA SALUd PÚBLICA DESPLÉS DE LA II GUERRA MUNDIAL}

\section{El Contexto de la Salud Pública en la Postguerra}

El fin de la II Guerra Mundial significó el triunfo de las fuerzas "democráricas" sobre las fuerzas materiales e ideológicas del colectivismo fascista. En ella perdieron la vida más de 40 millones de personas y se produjernn hechos que avergonzaron e indignaron al mundo civilizado. Como reacción social a estos hechos y al ejercicio totalitatio del poder. el valor de la vida humana adquirió nuevas dimensiones éticas y políticas en la sociedad occidental. Dimensiones que se expresan en nomas internacionales, actualmente vigentes. que no sólo consolidan el derecho a la vida, sino que reconocen un nuevo y más complejo derecho humano: el derecho a la salud. traducido como el derecho a una vida saludable y digna. Los Gobiernos del mundo. en la Declaración de Principios de la OMS (1947) y en la Declaración Universal de los Derechos Humanos (1948), reconocieron este nuevo derecho, así como las responsabilidades del Estado y de la sociedad civil en hacerlo efectivo. Derecho y responsábilidades que serían reiterados, por los Gobiemos del mundo. en la "Declaración de Alina Ata" (1978) y en la "Declaración Mundial sobre la Supervivencia. la Protección y el Desarrollo del Niño" (1990).

Se había iniciado, asimismo, un proceso de crecimiento económico sin precedentes en la historia de la humanidad que lamentablemente no significó, en los hechos, una mejor distribución mundial de la riqueza generada socialmente; aunque, ciertamente. contribuyó a que los gastos guberna- mentales en bienestar social adquirieran una rendencia creciente en la mayoría de países. Este crecimiento de la riqueza recién se agotaría en la primera mitad de la década de los serenta. cuando la economía mundial sufrió la crisis nús profunda y prolongada después de la Gran Depresión de 1930. En estas circunstancias de crisis, los Gobiernos del mundo adoptaron políticas de estabilización economica hasta ahora vigentes, que responden a una concepcion neoliberal del desatrollo. Aspecto importante de dichas políticas fue y es la reversión de las tendencias del gasto social gubernamental, que comenzó a disminuir de manera significativa.

Asimismo, en los años de ła II Guerra Mundial, el desarrollo de la tecnología había abierto el camino para el uso masivo por la Salud Pública de nuevos productos químicos contra los vectores, y de quimioterápicos y antibióticos contra los agentes biológicos; así como para la aplicación en la Medicina y la Salud Pública de los avances de la inmuncslogía. la virología, la genética y las ciencias de la conducta. En circunstancias de un proceso acelerado de urbanización que caracterizó a la postguera, esos progresos generaron una disminución significativa de la mortalidad y cambios importantes en los perfiles epidemiológicos mundiales. Por orra parte, en los países subdesarrollados la disminución de la moralidad se producía sin un decrecimiento de la fecundidad, y se iniciaba así la aceleración del crecimiento demográfico mundial que pronto se convertiría en un pro. blema internacional.

Paralelamente a esos procesos, se reinició la lucha material e ideológica por la hegemonía mundial entre Ins países capitalistas y socialistas que provocó confrontaciones militares en Corea (1950-1953) y en Vietnam (1970-1973). Posteriomente, el colectivismo del "socialismo real", arrastrado por la crisis económica mundial y sus propias contradicciones, se desplomó; el mundo asistió al derrumbe del Muro de Berlín (1989), la desintegración de la Unión Soviética y la disolución del Pacto de Varsovia (1991). Comenzaba así el dominio de los paises capitalistas y del individualismo neoliberal en el escenario mundial. En 1992. Carlyle Guerra de Macedo(") comentaba: "Muchos de nosotros henos recibido, con cierto sentimiento de alivio, el final del conflicto entre los ideologismos de Oriente y Occidente que an frecuentemente distorsionaron las maneras de percibir y comprender las realidades, y por lo tamlo de poder inervenir sobre ellas. Pero el Nuevo Orden que se estí conformando trae consigo orro tipo de ideologismo, quizás más peligroso no sólo poryue se plantea absoturamenre dominante y sin contradicción, sino porque prefende 
transformarse en otro tipo de ontologismo. Me refiero a la visión nooliberal del proceso de desarrollo (cuando se lleva a exrremos). La imposición de ese muevo ideologismo conlleva profundos riesgos...".

Hasta ese año en el campo de la salud. la concepción sociopolílica vigente en la Región, reconoxía a la salud como una necesidad básica y a su atención como un derecho y un deber de todos; más aún. el derecho a la salud tenía en casi todos los países de la Región el carácter de norma constitucional $\left(^{\circ}\right)$. Guerra de Macedo, en su calidad de Director de la Organización Panamericana de la Salud. cumplió con manifestar, entonces, su preocupación por comprender que la adopción de la visión neoliberal del desariollo, en una situación de crisis económica. significaría en la mayoría de los países de América Latina, una identificación acrítica de sus gobiernos con una concepción sociopolítica contraria a la que orientaba, hasta ese momento. la teoría y la práctica de la Salud Pública en la Región. No es difícil inferir que coincidía con Donabedian A. $\left({ }^{7}\right)$, cuando éste caracterizaba a la "posición liberal" como aquella que da preminencia absoluta al éxito personal y a la libertad individual frente a las fuerzas políticas, así como la compartida por los sujetos que orientan sus acciones, en el campo de la salud, a partir de las siguientes convicciones: la intervención del Estado traba las libertades individuales; existe una alta responsabilidad personal en el cuidado de la salud. porque toda recompensa debe ser merecida: la ayuda a los que no tienen éxito sólo se justifica por razones de caridad y filantropía: la única igualdad a reconocer es la que existe ante la ley. $Y$ que también habría coincididocon la opinión de $\mathrm{M}$. Bunge $\left({ }^{8}\right)$, sobre la filosofía que está detrás del ideologismo que ensalza al neoliberalismo: "es el individualismo tanno ontológica como noral. De acuerdo a él lu sociedudes un agregato de individuos autónomos. todos los cuales sono deberian ser libres. Esta visión es distinna a la realidad: la sociedad real es un supersistema compuesto de redes sociales y como tales tienen caracturisticas que sus componentes indiriduales carecen. Yel indiridualismo moral, esto es el egoísmo, es moralmente erróneo porque pasa por alto micstros deberes hacia los orros yor ende debilita las redes sociales aue sostiencu vicontrolem al agente individual".

Esas opiniones de rechazo al "individualismo" ontológico y moral, no significan la adhesión de sus autores al "colectivismo". Muy al contrario. son parte de sus argumentos para proponer una alternativa tanto al individualismo como al colectivismo. Bunge se cuida en explicitar lo siguiente: "El colectivismo es falso porque menosprecia la acción individual, y es moralmente erróneo por considerar al individuo como peón de las asi llamadas instancias superiores, tales como la raza. la nación o el partido. No es exrraño que el colecrivismo has a sido la filosofía detrás ranto de la derecha radicional (en particular el fascismo) y' el comunismo. (La mueva derecha es extremadamente individualista al extremo de que se llama asímismo libertaria)" $\left({ }^{8}\right)$.

Los hechos posteriores demostraron la validez de la preoupación de Guerra Macedo. En el escenario sudamericano de los siguientes años. se hizo evidente la identificación acrítica de las fracciones sociales dominantes con una visión neoliberal, en los términos caracterizados por Donabedian y Bunge. Ello explica el carácter de los esfuerzos que, durante la década de los noventa, efecuúan sus Gobiemos y parte de la sociedad civil para la construcción, a un alto costo social y de manera autoritaria, de las condiciones materiales e intmateriales para su concretización, es decir. para que eventualmente, en un escenatio futuro. considerado por ellos posible. esa visión se convierta en una realidad.

\section{La Salud Pública en la América del Sur de la Post- guerra}

En el contexto de las décadas cincuenta y sesenta, los responsables de la Salud Pública disponían de nejores medios legales, cognitivos y tecnológicos - que en el pasado para atender las demandas de la población. En esta nueva sillación, se produjeron importantes avances en el campo de la salud, sobre todo en la reducción de la mortalidad infantil, el control de las enfermedades transmisibles y en el desarrollo de la infraestructura sanitaria. Asimismo. en el curso de la búsqueda de una mayor racionalidad en la utilización de esta intraestructura se reconocio la importancia instrumental y valorativa de integrar las acciones de "reparación" con las de "promoción y protección" de la salud. Se comenzó así. a superar el viejo conflicto "atención curativa versus atención preventiva". con la percepción de la unicidad de la atención de un proceso unitario: la salud-enfermedad. Pronto, el concepto de "atención insegral de la salud", se constituyó en un tema básico en el discurso y la acción de la Salud Pública, y los términos de "recuperación" y "reparación" se incorporaron a los glosarios sanitarios. En 1952. el "Primer Congreso Interamericano de Higiene". efectuado en Cuba. define a la Salud Pública como "la ciencia y el arte de organizar y dirigir los esfuerzos colectivos para proteger, fomenar y reparar la salud" $\left({ }^{\circ}\right)$. Veinte años después. en Estados Unidos, la "Comisión Milbank para el Estudio de la Educa- 
ción Superior de la Salud Pública", presidida por el Profesor C. G. Sheps. define: "La Salud Prúlica es el esfuerzo organizado por la sociedad para promover proleger y reparar la salud de la población. Los programas, servicios o instituciones compromeridas enfatizan la prevención de la enfermedad y las ner esidades de la sulud de la población como un todo" $\left({ }^{10}\right)$.

En la segunda mitad de la década de los setenta, con el agravamicnto de la crisis económica, el incremento de la ingerencia del Estado en el financiamiento de la práctica de la Salud Pública y de otras vinculadas con el bienestar de la población fue detenido. Los Gobiemos sudamericanos adoptaron el modelo de desajoollo neoliberal. y la búsqueda de la "eficiencia" del Sector Público en Salud y de la Seguridad Social. supuestamente sobredimensionados, fue el argumento inicial con que sus gobernantes justificaron el recorte de los gastos públicos en salud. En 1987, en un Informe del Banco Mundial (") se lee: "En la mayoría de los países en desarrollo, el gasto publico en todos los sectores aumentó rápidamente durante los años sesenta y setenta, pero el lento crecimiento económico y los déficits presupuestarios sin precedentes de los ochenta ohligarnn a reducirlo... Ciertamente, es harto defendible la tesis en favor de un mavor gasto público en salud en los países en desarrollo, donde las expensas por este concepto de los sectores publico y privado, en conjunto, equivalen a menos del $5 \%$ de los gastos similares en los países desarrollados... Pero en la mayoría de los países la austeridad presupuestaria generalizada torna difícil la prédica en favor de un aumento del gasto público...".

Los autores de ese Informe, partiendo de la supuesta imposibilidad de aumentar el muy insuficiente gasto públicoen salud, se plantean la pregunta "¿Qué puede hacerse?". Para dar su respuesta, con base a una racionalidad instrumental. señalan 3 problemas: (a) gasto insuficicnte en salud e ineficacia del gasto en función de costos, (b) incficiencia interna de los programas públicos. y (c) desiguaidad en la distribución de los beneficios derivados de los servicios de salud. Asimismo, proponen 4 reformas: (a) cobro de aranceles a los usuarios de los servicios públicos de salud. especialmente para medicinas y atención curativa; (b) esrímulo de programas bien diseñados de seguro de salud para ayudar a movilizar recursos hacia el sector de la salud; (c) estímulo al subsector privado para que provea servicios por los cuales los consumidores estén dispuestos a pagar; (d) descentralización de la planificación. presupuestación y adquisiciones para los servicios de salud gubemamentales, particularmente aquellos que rinden beneficios privados por los cuales se cobra a los usuarios. Para justificar y aplicar las reformas propuestas, clasifican los bienes y servicios provistos por el sistema de salud en dos categorias: "bienes netamente privados". cur yos beneficios son captados totalmente por la persona que recibe el servicio de salud. y "bienes puramente públicos". cuyos beneficios perciben por igual todos los miembrus de la comunidad. Luego, aceptando que esta clasificación y las siguientes comparaciones no son exactas, consideran a los primeros bienes como idénticos a los servicios de "utención curativa", por cuyo uso el público tiene una mayor disposición a pagar. y equiparan a los segundos con los de "atención prevenriva". La conclusión es obvia: se debe reducir la responsabilidad del Gobierno en cuanto al financiamiento de los "bienes netamente privados", por ser éstos de beneficio sólo personal ( $\left.{ }^{11}\right)$.

Cinco años después, el mismo Banco Mundial en su "Informe sobre el Desarrollo Mundial 1993: Invertis en Salud". recuerda que la teoría económica sugiere que, contrariamente al caso de otros sectores de la economía, los gobiernos deben intervenir en el Sector Salud por la presencia de defectos del mercado, sugerencia que se fundamenta en la evidencia de que las acciones aisladas de los productores y consumidores no permiten resultados socialmente óptimos o económicamente eficientes. Precisa, además que las principales tareas gubemamentales deben ser la regulación de las deficiencias del mercado y el financiamiento eficiente de la atención de los pobres. Asimismo, recomienda para los "países de ingreso mediano", dos políticas de "gran imporrancia": (a) reducir el gasto público en hospitales, formación de especialistas y servicios discrecionales; y (b) alentar la financiación y la oferta privadas de seguros para todos los servicios clínicos discrecionales. Por último. a partir de la supuesta validez de sus argumentos sobre la dicotomización de la producción sectorial en "bienes públicos" y "bienes privados, diferencia dos formas de interacción entre los servicios de salud y la población: "programas de salud pública", orientados a la colectividad y financiados por ésta, y "servicios clínicos", orientados a dar respuesta a la demanda de los individuos enfermos y financiados, total o parcialmente, por éstos $\left({ }^{12}\right)$.

Esa dicotomización parte de la negación de la unicidad de la "atención integral de la salud" para retornar, con fines económicos, a una concepción superada en la década de los cincuenta. Lo que se traca es de justificar y, luego, formalizar la existencia de dos sub-sistemas paralelos: el de la "Salud Pública estaral", encargado de la prestación de los servicios preventivo promocionales y de la producción de otros servicios básicos para la atención de la demanda 
social de la pohlación de pobres; y el de la "arención méclic a". encurgado de la producción de servicios curativos personales para la alención de la demanda cconómica del resto de la población.

En el año 1906. la totalidad de los gobiemos de los países de Amćrica Lalinat informaron estar instrumentando una reforma de sus sistemas nacionales de salud, con el apoyo financicro del Banco Mundial. Las concepciones y las políticas de reformatilizadas por estos países eran. con algunas variaciones, las recomendadas por el Banco en 1987 y $1993\left({ }^{13}\right)$.

\section{LA SALUD PÚBLICA EN UNA SOCIEDAD EN CRISIS}

\section{La Sociedad al Final del Segundo Milenio}

En la teoría de la modernidad de J. Habermas, se argumenta que la dinámica de las sociedades modernas se caracteriza por presentar algunas tendencias generales como: el desenganche entre el mundo de la vida y las estructuras de reproducción económica y del poder que se autonomizan y se configuran como sistemas autoregulados, así como el sometimiento del mundo de la vida por el sistema global social. En este contexto. las formas de coordinación intersubjetiva de la acción social ceden espacio y. en gran medida, quedan subyugadas por las formas instrumentales y estratégicas de conodinación que los sistemas económico y polícicorealizan. Es decir, las acciones orientadas al entendimiento y que utilizan como criterios de racionalidad la consistencia moral y el asentimiento consensual retroceden y quedan subordinadas a las acciones orientadas al éxito material que utiliza como criterios de racionalidad la rentabilidad económica y el dominio político. Las acciones orientadas al entendimiento tienen una racionalidad garantizada en las tradiciones culturales y los valores éricos internalizados en la colectividad. y' utilizan como medios de coordinación y control social la influencia del prestigio y el compromiso valorativo. De manera muy distinta. las acciones orientadas al éxito material tienen una racionalidad garantizada en lá riqueza y en la coerción, y utilizan como medios de coordinación y control social el dinero yel poder (', s,14).

Al final de este milenio. los hechos están validando la tecría de modernidad de Habermas. Estamos viviendo en un nuevo mundo donde coexisten. por un lado procesos de globalización y concentración del dinero y del poder: y por otro, procesos de profundización de desigualdades entre los hombres y de globalización de la desocupación. la pobreza y la violencia. Mundo que desde la segunda mitad de la década de los setenta está sometido a una profunda reorganización para consolidar estructuras económicas y políticas que, accualmente, recrean sociedades que marginan y acostumbran, al hacerla cotidiana, a la marginación. así como devalúan las tradiciones culturales. En el centro de tal reorganización está el proyecro de desartollo neoliberal, con su concepción individualista del ser humano, su racionalidad instrumental y sus propuestas de integrar la sociedad en función del "libre mercado", a través de una mercantilización y privatización de todos los procesos sociales que puedan ser compatibles con los intereses privados y una moral egoísta y pragmática. Por el alto costo social de la instrumentación del proyecto, su legitimación es un aspecio esencial de esta reorganización. En este sentido, por un lado. se argumenta que dicho proyecto es el único camino viable para el "descurrollo del capital humano y del capital social" en el largo plazo; y por otro. se trata de inducir a un cambio de los valores intemalizados y de las normas vigentes en la socjedad, para que éstos legitimen - antes que a las demandas sociales para satisfacer necesidades actuales de la población - a los requerimientos materiales del proyecto neoliberal.

\section{La Reforma de los Sistemas de Salud al Final del Segundo Milenio}

Al final del segundo milenio, en ese nuevo mundo y específicamente en los países de Amćrica del Sur, la política de salud está tocalmente subordinada a la política económica, que se ha constituido en el eje central de los proyectos nacionales de desartollo. Además, el Banco Mundial, entidad financiera, comanda la asignación de recursos de la Cooperación Externa en el campo de la Salud y se ha converido en el principal referente teórico de las reformas de los sistemas nacionales de salud. Por ende. Ios Ministros de Economía lideran la acción gubermamental y los economistas imponen su saber, de manera autoritaria, en los sectores sociales y en la práctica de la Salud Pública. Liderazgo y autoritarismo que someten a una debilitada autoridad política sectorial; no consideran los saberes de otros profesionales, e ignoran la racionalidad y la visión de otros actores sociales que actúan en el campo de la atención integral de la salud.

En esa situación, nadie puede negar que los procesos de reforma del sistema de salud en los países de la Región se 
realizan en función de la lógica económica de una reforma del Estado que está privatizando sus estructuras y procesos para adecuarse a los requerimientos del proyecto neoliberal, bajo la tutoria de organismos financieros intemacionales. No puede sorprender entonces. que se siga recortando los alcances del derecho a la salud. pariendo de la premisa de la imposibilidad o inconveniencia de incrementar el gasto público en salud - históricamente insuficiente en América Latina - y se insista en que Ias autoridades sectoriales de salud deban limitarse a reformas orientadas a garantizar el mejor uso de los escasos recursos existentes y a incrementar el apoyo financiero no gubernamental. Para esto último, se están creando las condiciones para convertir al Sector Salud como un ámbito directo y legítimo de lucro y acumulación económica. antes que un campo de satisfacción de una necesidad básica y de acumulación del bienestar y la calidad de la persona humana.

En el discurso oficial de las autoridades políticas a cargo de la conducción de los procesos de reformas comentados, se insiste en que tales cambios están orientados hacia el logro de mayores niveles de eficiencia para una mayor efectividad, equidad y calidad en la atención de la salud. Sin embargo, la evaluación de los resultados de esos cambios muesuan que cuando se ha logrado aumenta la eficiencia tecnica de la atención, los efectos más persistentes son: el incremenlo de las desigualdades en el acceso a los servicios de salud de calidad y la mayor insatisfacción de los usuarios por la efectividad y la calidad de dichos servicios. Además, pocas veces se verifica un incremento de la eficiencia en la asignación de los recursos de salud. Kutzin J. ( ${ }^{15}$ ), informando sobre los resultados de un estudio realizado por la Organización Mundial de la Salud afirmaba: "La evidencia presenrada en este informe indica que los resultados de las reformas al financiamiento y a la organización del Sector Salud están influenciados pormumerosos factore's. Las decisiones de las auroridades gubernamemales del Secror pueden afectar a algunos de los factores inmediatamente. miemras otras decisiones tendrám un efecto solamente en el mediano y largo plazo. Sin embargo, ciertos factores contexmales que afecin las consecuencias de la refonna, rales conno el componamiento mucroeconómico, desarrollo de la infraestructura, niveles educativos, y momas culturales, esrán fuera de la influencia de un Ministro de Salud. Como un resultado. políticas similares pueden tener muy diferentes consecuencias en diferentes ambientes...".

En nuestro entender, la explicación de los resultados insarisfactorios de las reformas en la Región se encuentra además de los factores informados por Kutzin - en las restricciones impuestas, en el curso de la búsqueda preferente de la eficiencia, a la universalidad, la equidad. la integralidad $y$, especialmente. la democratización de la atención de la salud. Restricciones derivadas de la aplicación unilateral de una lógica de la acumulación económica y, en consecuencia, de una racionalidad esencialmente instrumental.

Los actores sociales teñidos con el "ideologismo" neoliberal, están convencidos de que los servicios de salud son "mercancias" y que el "libre juego de la demanda! ofera" es, en el largo plazo, "el mejor mecanismo para regular la producción y' el consumo equirativo" de dichas mercancías. Por ello, cuando comentan esos resultados y el alto costo de la reforma, no los atribuyen al núcleo económico de ésta, sino al supues(o hecho de que no es suficientemente liberal. En consecuencia. no buscan nuevos caminos basados en el consenso interdisciplimario. sino que persisten en reelaborar normas jurídicas y redefinir conceptos tecnológicos y preceptos éticos, con el fin de construir y hacer hegemónico, en el mundo cotidiano y en el mundo académico, un marco conceptual normativo, de acuerdo con el cual sería imposible o incorrecto pensar y argumentar en favor de propucstas contrarias a una racionalidad que entiende al beneficio económico como un fin en si mismo. En este escenario ideologizado. los conceptos de solidaridad, igualdad y justicia social, que emergieron en la modernidad. son redefinidos o dejan de estar presentes en el espacio de discusión pública y ceden lugar. también redefinidos, a los de eficienciá, equidad, calidad, libre elección y competividad, entendidos éstos como condiciones. necesarias para una supuesta modernización, otroconcepto sometido a una redefinición. que pretende entenderlo como equivalente al de privatización. Tudos los conceptos y todas las normas que no corresponden a las ideas neoliberales son considerados como "obsoletos" o "uradicioncles" y tienen que ser abandonados o redefinidos. Para estos liberales, la creciente marginación o postergación de los "servicios clinicos" o "servicios de cuidado de salud personal". de gran pane de la población, puede ser hatsta lamentable peroes un asunto de responsabilidad individual y. por ende, no liene ni debe ser considerado un "compromiso social", ni mucho menos un derecho social y juridico, en el proceso de construcción de una economía de mercado que es visualizado, por ellos, como el únicocamino viable para el desartollo humano.

\section{Comentarios Finales}

En América del Sur, los resultados insatisfactorios de las reformas de los sistemas de salud siguen siendo justi- 
ficados por las autoridades políticas, con la afímación de que son temporales y parte de un costo social ineludible para instrumentar el proyecto neoliberal, único camino viable para el desarrollo humano. Sin embargo, cada vez es más difícil argumentar la valide $z$ de la parte esencial de esta afirmación. Hay scñales que la sostenibilidad y el crecimiento del sistema neoliberal globalizado están amenazados por las crecientes desigualdades, la devaluación de principios morales y jurídicos surgidos en la modernidad, el marcado desequilibrio cntre los poderes mundiales, y el aumento de la población. La mayor parte de especialistas en el tema, coinciden en sostener que dicho sistema no podrá continuar con su evolución actual y los más radicales, consideran que se desplomará si no elimina o controla esas amena$\operatorname{zas}(16.17,18.19 .20)$.

Se reconoce que el mundo actual está dirigido por inversionistas internacionales y corporaciones transnacionales que buscan rendimientos de corto plazo, que hacen tratos con gobiernos cuya única prencupación es la de crear empleos en su país y logra el status de productores de categoría mundial, sin importarles que las remuneraciones de sus trabajadores sean cada vez más insatisfactorias. Esto da lugiar a una sobrecapacidad productiva en muchas economías nacionales, sin que de manera simultánea se creen nuevos mercados en los países receptores de esa producción. Los imperativos con respecto al rendimiento de las empresas transnacionales en un mundo competitivo, con mercados que se reducen con relación a una sobreproducción. hacen que los sueldos en los países subdesarrollados se mantengan bajos. por lo que los trabajadores no tienen dinero suficiente para comprar lo que producen.

Los Gobiernos han sobreestimado los beneficios de los mecanismos del libre mercado. así como la validez del saber actual de la cienciá económicá. I a autorregulación de loss mercados es una teoría que ha probado ser equivocada. "Las des. igualikudes producidus por los mercudos produjeron un desequilibrio en el desarrollo económico que a su vez. provocó que cientos de millones de personas se sientan acorraladase impotentes. De aruerdo con el Institute for Policy Studies de Washington. en la actualidad sólo 14 países en desarrollo que privilegiaron el libre mercudo han logrado imprimir dinamismo a su crecimiento económico, mientras que en 140 más éste se rédujo, se estancó o se revirtió" $\left.{ }^{21}\right)$. La ciencia económica se ocupa hoy de la economía como un subsistema de la sociedad y prescinde de las cuestiones de legitimidad; desde esta perspectiva parcial reduce los problemas de racionalidad a consideraciones de equilibrio económico y a cuestiones de elección instrumental. Por tal razón, esta ciencia muestra su imprecisión para hacer pronósticos: "Durante 25 años quienes elaboraron aquellos en Estados Unidos no lograron detectar cuatro de cinco recesiones" $\left({ }^{21}\right)$.

El capitalismo fortalecido que surgió tras el colapso de la Unión Soviética no ha producido ninguna ideología orientadora sino un "ideologismo" neoliberal que, asumiendo "el penoso realismo del mercado", privilegia un individualismo egoísta y promueve la avaricia y la prepotencia de los "triunfadores". "El principio érico que directamente justificaría la distribución de la renta en una sociedad libre de mercado es el siguiente: a cada uno, según lo que él y los instrumentos que él posee producen" (Friedman, Milton. "Capitalismo y Libertad", 1962). En un mundo donde el empleo adecuado es cada vez más escasoe inestable, el trabajador debe soportar el recorte de sus derechos para disminuir los "sobrecostos laborales" que, supuestamente, limitan la competitividad de la empresa. En la perspectiva de los países subdesarrollados y de los trabajadores ha surgido el "globalirarisno". Un ejemplo de ello, es el intento de algunos países poderosos de aplicar sus legislaciones nacionales en el plano internacional $\left({ }^{21}\right)$

Se estima que la población mundial se duplicará entre 2030 y 2040 , correspondiéndole a los países subdesarrollados las mayores tasas de crecimiento demográfico. Actualmente, se estima que $80 \%$ de la población del planeta que vive en las economías subdesarrolladas consumen apenas el $25 \%$ de los recursos y de la energía mundiales, en tanto que los países desarrollados. que concentran la riqueza mundial y el $20 \%$ de la población, consumen el $75 \%$ de dichos recursos y produce gran parte de los desechos industriales, tóxicos y domésticos de todo el mundo. Por tanto, al excedente de producción se agregará el de mano de obra. en un escenario de empobrecimiento social y degradación ecológica. Situación que aumentará las tensiones económicas y sociales que, a su vez, incrementarán las dificultades para la coordinación y control social $\left({ }^{21}\right)$.

En las circunstancias del replanteamiento del proyecto de desartollo actual mente dominante. cualquier propuesta de reforma de la Salud Pública - con pretensión de eficacia, corrección y veracidad - tiene que ser concordante, a nuestro entender, con las siguientes consideraciones:

2. Los derechos humanos y, en particular, Ias exigencias internas de libertad, justicia y solidaridad se mantienen como valores éticos supremos de la moralidad que contienen y expresan la dignidad humana. Los principios de la metanorma moral (moralidad) han de ser traducidos a normas morales concretas en un contexto históri- 
co social dado (eticidad) mediante la deliberación práctica. Se acepta que esta tradución es faliblẹ. por consiguiente, ha de realizarse a través de una deliberación conjunta y leal entre sujelos. en el curso de la cual se pueden comprobar la validez de las diversas propueslas normativals.

Una de estas propuestas, considerará que una racional atención de la salud de la población, en el marco de los derechos humanos, debe cumplir ks siguiente requisites:

- universalidad y lihertad en el acceso a Ios servicios de salud;

- equidad en la asignación de los recursos y en la distribución los servicios de salud:

- integralidad en la organización y prestación de servicios de salud;

- eficiencia técnica en la gestion y uso de los recursos asignados;

- solidaridad soxial y generacional en el financiamiento de la atención de la salud; y

- caŕcter participatorio, para garantizar el cumplimiento de los requisitos anteriores.

2a. El Estado es un conjunto de institucinnes sociales que establece un orden en un territorio determinado que respalda con una garantía coercitiva centralizada. El Estado y el mercado son necesarios para la socicdad y ambos son recíprocamente dependientes y complementarios. Si bien la actividad económica es imposible sin un malco institucional estable, una sociedad con norcados débiles carece de dinamismo econónico. Además. en la actualidad. las políticas globales y sectoriales necesitan una nueva forma de interacción de los actores públicos y privados en los diversos ámbitos.

$3^{a}$. Desde un punto de vista normativo, un objetivo principal del Estado es lograr la integración del conjunto de la sociedad en el desámollo. en el sentidu más amplio del concepto. El pacto social que origina toda la institucionalidad pública sólo tiene sentido como un factor de mejoramiento del conjunto social. De lo anterior. se infiere la preocupación particular por cluienes son menos favorecidos por el libre juego de las fuerzas del mercado. En consecuencia. corresponde a una institucionalidad democrática inıpedir la captura del Estado por intereses corporativos o sectoriales y mantener una clara separación entre los intereses generales y los particulares. Un papel esencial de la institucionalidad estatal es el de asegurar el Estado de Derecho en gene- ral. Al mismo tiempo, uno de los aspecoss institucionales más importante es el de la regulación del mercado.

4". El desamollo humano requiere de procesos pulíticos y sociales gubernables, es decir, que tengan un curso preestablecido para la articulación de propósilos y la resolución de contlictos. Los programas de reforma y los consecuentes cambios institucionales. así como las propuestas para anticipar situaciones críticas, precisan de un apoyo sostenido en sus diversas fases. Para ello se necesitan esfuerzos permanentes para analizar, en conjunto. los argumentos de los distintos actores sociales y phantear opciones jerarquizadas de políticas de consenso. eficaces y eficientes. Es decir, se necesita definir acciones políticas de carácter comunicativo. en los términos definidos por Habermas.

5. Los procesos de concertación, como propios de unáación comunicativa, suponen la existencia de una scric de factores en cuanto a la participación leal y responsable de los actores sociales en la elaboración y toma de decisiones de políticas públicas. Por otra parte, los acuerdos de concertacion deben procesarse institucionalmente, para evitar que las instituciones democráticas queden reducidas al papel de instancias de mera ratificación de lo acordado. Junto al impulso de los acuerdos. se requiere generar escenarios que permilan encausar y negociar conflictos e intereses contradictorios; de otro modo el consenso se puede converir en su propio enemigo al intentar reemplazar la dinámica social por las negociaciones de las cúpulas. Para que la concertación se considere como un ejcricicis legítimo y conveniente, debe atender a los diversos intereses y partes: en este sentido conviene más regular y negociar que ignorar los grupos de presión.

$6^{3}$. I_a "salud" y la "enfermedad" son fenómenos inherentes a la vida misma. La muerte es la negación de ambos. Sin salud no hay seguridad de la supervivencia; no hay posibilidad de desamollar capacidades y potencialidades productivas y sociales; no hay posibilidad de participar activamente, como individuo y como ser colectivo, en la vida social; no hay libertad ni ciudadanía plena. La salud humana es, así, una necesidad básica de carácter biosocial que va más allá del planteamiento de la salud como un derecho sancionado jurídicamente.

$7^{a}$. La definición de políticas especíticas concertadas para tratar problemas de alta complejidad, como son las que enfrenta la Salud Pública, exige la existencia previa de políticas generales y sectoriales concertadas que sirvan de marco de referencia para un trabajo interdisciplinario 
en esa área específica, con la participación de todos los actores sociales relevantes. Los responsables de este Irabajo, una vez que estén ubicados y contextualizados, deberán buscar, con un enfoque prospectivo y dentro del marco polícico general y sectorial concertado, opciones de respuestas racionales a una serie de interrogantes sobre los diversos problemas del conocimiento y de la acción en las distintas dimensiones de dicha área. El ordenamiento sistemático de dichas respuestas constituirá una Agenda de Trabajo para llegar a acuerdos sobre "lo que es necesario y posible hacer" en el país para el desarrollo efectivo de la Salud Pública.

Con relación a estas últimas consideraciones, nos parece importante citar al pensador Paulo Freire, cuando decía "Como un ser inconcluso y consciente de su inconclusión, el hombre es un ser de la búsqueda permanente. No podría haber hombre sin búsqueda, de la misma forma como no habría búsqueda sin mundo... esta bísqueda implica un sujeto, un punto de partida, un objerivo.. Nadie puede buscar solo. Toda biisqueda en aislamiento; toda búsqueda que se haga por intereses personales o de grupo, necesariamente es buisqueda contra los demás. Solamente en conumión es auténtica".

\section{BIBLIOGRAFÍA}

1) Bunge M. Filosofía de las Ciencias y Técnicas Sociales. I Curso Internacional de Filosofía de las Ciencias y Técnicas Sociales. Escuela de Postgrado de la Universidad de Lima. Lima: 1996.

2) Bustios C. Salud Pública: Notas y Reflexiones. Escuela Nacional de Salud Pública. Lima: 1996.

3) Ilabermas J. Tcoría de la acción comunicativa. Il. Crítica de la razón funcionalista. Buenos Aires: Taurus; 1990.

4) Habermas J. Teoría de la acción comunicaliva. I. Racionalidad de la acción y racionalización social. Buenos Aires: Taurus Ed; 1989.

5) Guerra de Macedo C. El Contexto. En: OPS/OMS. ed. La Crisis de la Salud Pública. Publicación Cicntífica N $^{\circ} 540$. WashingIon DC 1992. pp. 238.
6) Fuenzalida-Puelma If, Schole S (Compiladores). El Derecho a la Salud en las América. Estudio constitucional comparado. Publicación Científica 509. Washington DC. OPS, 1989.

7) Donabedian A. Aspects of Medical Care Administration Cambridge: Harvard University Press; 1973. p. 4-15.

8) Bunge $M$. Tecnoholodemocracia. Una Alternativa al Capitalismo y Socialismo. Revista Peruana de Filosofía Aplicada $N^{\circ} 4$. UNMSM. 1997 p. 13-14.

9) Primer Congreso Interamericano de Higiene. Relato oficial sobre organización e integración de los servicios de salubridad. La Habana, Cuba.1952.

10) Milbank Memorial Fund Comission. Higher Education for Public Health. A Report of the Milbank Memorial Fund Commission. 1972. p. 3

11) Banco Mundial. El Financiamiento de los Servicios de Salud en los Países en Desarrollo. Una Agenda para la Reforma. Bol Of Sanit Panam 1987; 103(6): 695-708.

12) Banco Mundial. Informe sobre el Desarrollo Mundial 1993. Invertir en Salud. Banco Mundial.Washington, DC.1993.

13) OPS/OMS. Progreso de las Actividades de Reforma del Sector Salud. Documento CE $118 / 11$ (Esp). $118^{*}$ Reunión del Comité Ejecutivo del Consejo Directivo de la Organización Panamericana de la Salud. Washington, DC. Junio de 1996. p.5-9

14) Uribe FJ. Agir Comunicativo e Planejamento Social (Uma Crítica ao Enfoque Estratégico). Río de Janeiro. FIOCRUZ. 1995.

15) Kutzin J. Experience with Organizational and Financing Reforcement of the Health Sector. Current Concerns SHS Paper number 8. Division of Strengthening of Health Services. World Health Organization. 1995 Jan: 41

16) Sutherland P. Beyond the Market, A Difrerent Kind of Equity. IHT Feb 20: 1997.

17) Greider W. Onc Word. Ready or Not-The Manic Logic of Global Capicalist. Nueva York: Simond and Schuster: 1997.

18) Forrester V. O Honror Económico. UNESP. Librairie. Arthéme Fyardl, 1996.

19) Shapiro RB y Ceo M. Growth Trough Global Sustainability. HBR. 1997.

20) Thurow LC. The Future of Capitalism-How Today's Economic Forces Shape Tomorrow's World. Morrow: 1996

21) Machado F. Administración eficiente de la innovación tecnologica en los países en desarrollo. Comercio Exıerior - México. 1998; 48 (8): $607-16$. 